\title{
Impacted canines-literature review
}

\author{
D. Brézulier ${ }^{1}$, O. Sorel ${ }^{2}$ \\ 1 Qualified Specialist, Hospital-University Assistant, University of Rennes 1 \\ 2 Qualified Specialist, University Professor-Hospital practitioner, University of Rennes 1
}

Our review, as of 1968, proposed a bibliographic analysis on the subject of impacted canines. The considerable number of studies on this subject, as well as the journal's issues devoted to this subject, prove that the diagnosis, the therapeutic choice, and finally the setting up of this tooth, remains a delicate problem. We propose here, if possible, a comparison of the techniques used in 1968 with those used nowadays.

\section{DEFINITION}

Alain Benauwt and Claude Duchâteau use four qualifiers for canines that have not erupted: impacted, enclaved, disintegrating, and ectopic. An impacted canine is defined as, "a tooth retained in the maxillary or mandibular arch beyond the date of eruption, surrounded by its pericoronal sac and has no contact with the oral cavity" 15 . The orthodontic dictionary has two defini- tions: The term impacted tooth is defined as a mature tooth whose follicular sac is not in contact with the oral cavity. The retained or enclosed tooth is a mature tooth whose eruption has stopped, the follicular sac being in contact with the oral cavity. The incidence of impacted maxillary canines fluctuates between $0.8 \%$ and $2.8 \%$, and it is higher in women $8,18,29$

\section{BACKGROUND}

Seven references are quoted by Benauwt and Duchâteau. Let us note that the basic principle regarding the removal of the bone covering the tooth was already known in 1539! The following authors (Talbot, Angle, Dunn, Tucail, Monteil) set up a traction device. What needs to be analyzed are the means of retention of this device to the enamel: they are either sealed or rest on the sealing of a brace. Some techniques require a perforation of the canine. These available devices, which could now be described as

Address for correspondence:

Damien Brézulier - 2, Place Pasteur - 35000 Rennes

E-mail: damien.brezulier@me.com

Article Received: 11-08-2016. Accepted for publication: 11-08-2016.

This is an Open Access article distributed under the terms of the Creative Commons Attribution License (http://creativecommons.org/licenses/by/4.0), which permits unrestricted use, distribution, and reproduction in any medium, provided the original work is properly cited. 
obsolete or even iatrogenic, remind us of the progress of the collaborative tech- niques in dentistry in the second half of the $20^{\text {th }}$ century.

\section{ETIOPATHOGENESIS}

Our authors distinguish the local causes from the general causes. Although they remain topical, they have since then been supported or clarified in the literature.

\section{Local Causes}

Beyond the factors mentioned by the authors, one must mention the decisive aspect of the space available for the canine on the arch and also the maxillary morphology22,25,41.

In fact, a maxillary endognathia often leads to the retention of canines which, at best, erupt in an ectopic location, and at worst, remain impacted. Moreover, the mesialization of the posterior sectors can lead to a lack of space for the development of the canine.

Of course, any situation that presents and obstacle for the eruption of the permanent canines, can lead to them becoming impacted: cyst, tumor, supernumerary tooth, persistence of the temporary tooth, and

\section{SYMPTOMALOGY}

The discovery of the canine evolutionary anomaly is most often discovered during the clinical examination. As described by Benauwt and Duchateau, persistent temporary teeth or diastema after their exfoliation infection ${ }^{3}$. Also note the iatrogenic origin associated with impacted teeth due to early extraction of temporary canines.

Some authors explain that the absence of guidance because of the absence of lateral incisors, by loss or agenesis, or anomalies in shape or in the position of these teeth leads to a defective positioning of the canines ${ }^{6,33}$. This guiding theory is underreported by the fact that many cases of impacted canines have completely normal lateral incisors in form, volume, and position ${ }^{32,35}$.

\section{Regional, general, and systemic causes}

Regional, general, and systemic causes are now better understood. Diseases such as hypothyroidism, cleidocranial dysostosis, imperfect amelogenesis, Down's syndrome, and osteoporosis can be associated with impacted canines. The genetic factor is currently being studied for cases of impacted canines ${ }^{34}$.

should draw our attention. The position of the canine can be evaluated via palpation. Moreover, a Quintero sign may lead to the suspicion of a problem with the development of the canines. 


\section{RADIOLOGICAL RESEARCH}

In 1968, three types of imaging were used to specify the position of the impacted canine: a retroalveolar x-ray, an occlusal film, and a three quarters or profile image. Today, the orthopantomogram, the basic snapshot of the dental checkup, makes it possible to highlight many cases of canine impaction (Fig. 1). Different parameters can be measured to establish the risk of dental impaction ${ }^{1,16}$.

It should be noted, however, that the panoramic image is a computerized tomography (CT) image. Therefore, a tooth that would be at a distance from the cutting plane (palatal or symphyseal canine) may not be visible on the images (Fig. 2).

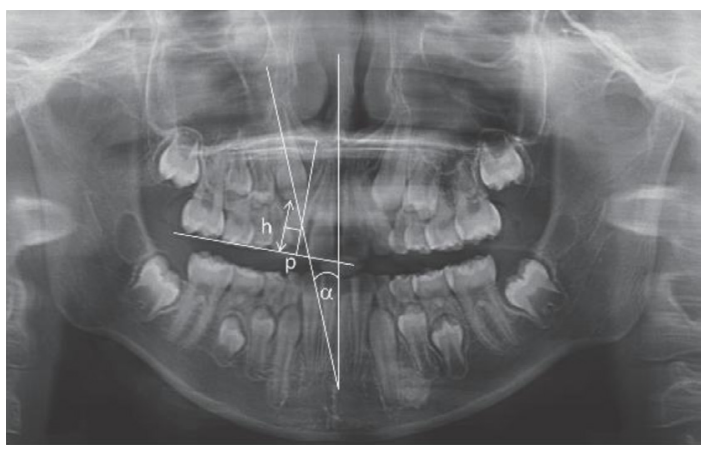

Figure 1

In this 9-year-old patient, the panoramic analysis shows a risk of canine impaction. Three measures are taken into account to establish the risk of dental impaction: the angle between the axis of the tooth and the interincisor line (a), distance to the occlusion plane (h), and finally the projection of the canine tip onto the lateral incisor (p). The tables of Ericson, Kurol, and Baccetti estimate the percentage of impaction in relation to this data.

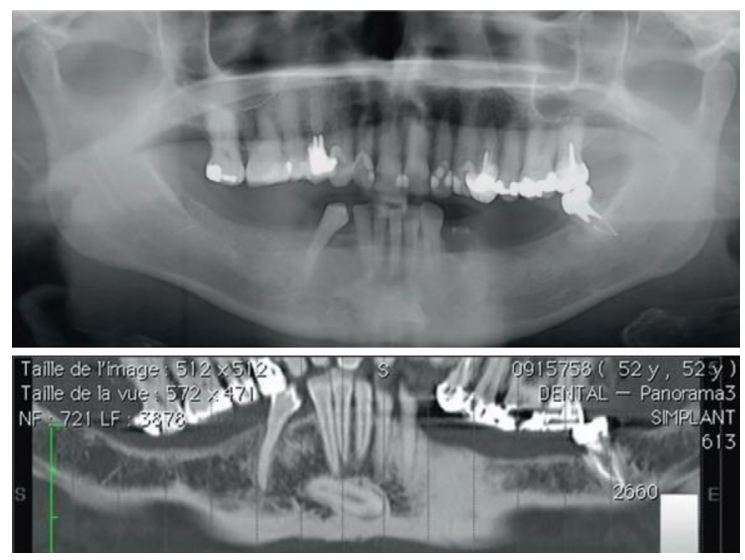

Figure 2

The orthopantomogram is a tomographic scan. Therefore, a tooth outside this cutting plane is not visible on the image. On the panoramic view, the mandibular impacted canine can go unnoticed, as is shown in the image. The contribution of the CBCT confirms the presence of an ectopic canine. In addition, relationships with mandibular incisors and cortical bones can be precise ${ }^{38}$. 
In this context, the accuracy of the information from these images is no longer sufficient in complex situations. The use of the cone-beam computed tomography (CBCT) thus have several objectives: beyond the simple evaluation of the risk of impaction (KPG index) ${ }^{13,14,23,24,36}$, it will make it possible to objectify the relations with the adjacent teeth (roots of the lateral incisors, apex of premolars) ${ }^{17}$. Depending on the complexity of the treatment, the decision of the most appropriate and least risky traction pathway to set up the canine can be made objectively ${ }^{19}$ (Fig. 3).

\section{TREATMENT INDICATION}

It seems illusory today to want to determine an age limit for canine placement. However, note that after the age of 20 years, the rate of failure for the traction increases drastically ${ }^{5}$.

\section{Treatment, Pretherapeutic stage}

We still think it is relevant to address a child who appears to be suffering from a growth disorder to his or her attending physician or the endocrinologist. It is clearly shown that several endocrine disorders or hormone treatments modify dental maturation. The holistic vision of the patient is necessary: it allows us to identify causes that are sometimes far removed from our odontocentric concerns.

\section{Treatment, Surgical step}

\section{Surgical phase associated with orthodontics}

The surgical approach for the impacted tooth will depend on its situation: palatal, vestibular, or mixed. In the first step we will detail the techniques used for the palatal teeth. There are two opposing techniques: open techniques and closed techniques, which, according to the literature, are not distinguished in terms of the results obtained.

The "open" technique consists of exposing the tooth surgically and then placing a covering bandage over the wound for 10 days. The tooth can then erupt. As soon as it is sufficient, an

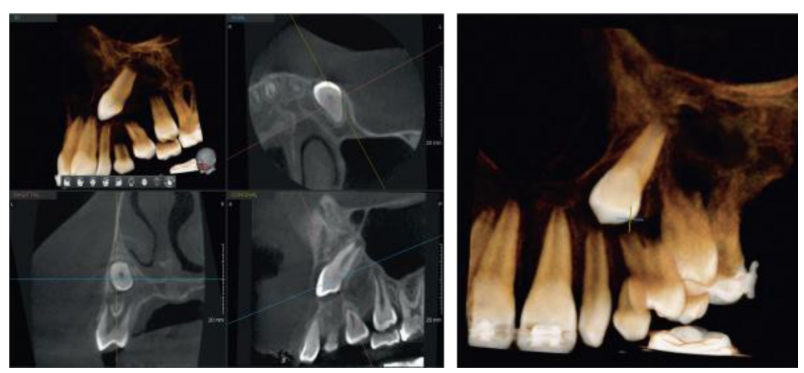

Figure 3

CBCT allows the practitioner to represent the position of the canine in space. The latter is located in relation to the other teeth. On the left scan, the canine appears at the level of the palatal root of the premolar. 
attachment is attached to the crown to allow the positioning of the tooth on the $\operatorname{arch}^{26}$. Of course, the deeper the canine, the more significant an obstacle it is and the more it hinders an open traction. The main advantage of this technique lies in the palatial evolution of the crown, thus tending to isolate the apex of the canine from the adjacent teeth ${ }^{28}$. It is also easier to follow the evolution of the coronal movement when the tooth is visible.

The second technique, called the "closed" technique, consists of an initial surgery on the tooth, the setting up of a traction device, and then the repositioning of the palatal flap. This technique requires planning the tooth's traction path to minimize the risk of collision with adjacent teeth ${ }^{4}$. This method has the advantage of decreasing the morbidity compared to the open technique. However, there are many disadvantages: preoperative bonding difficulty, detachment, tensile failure, and traction imprecision ${ }^{2}$ (Fig. 4).

Comparing these two techniques is tricky. At the moment, there is no data available to prefer one of the two in terms of periodontal health, esthetic results, intervention time, and patient perception ${ }^{30,31}$.

With regard to vestibular teeth, the approach will depend on the situation of the tooth: vestibulolingual and mesiodistal position of the crown, amount of gingiva attached and the position in relation to the mucogingival line. There are three types of intervention: operculization of the vestibular gingiva, an apically placed flap, and a closed technique 27,40 .

The apically repositioned flap is used if the pulled tooth is likely to be in the free gingiva or if the crown is in a mesial position compared to the lateral position $^{38}$. The closed technique is used if the canine has a central position on the crest and/or is apical to the mucogingival line (Fig. 5).

\section{Terminal Surgical Phase}

This involves the self-transplantation of the canine. This technique can be used if no other therapeutic options are indicated or if they are unsuccessful. It allows for the rapid treatment of edentulous situation, but it requires an orthodontic and surgical preparation at the recipient site $^{21}$.
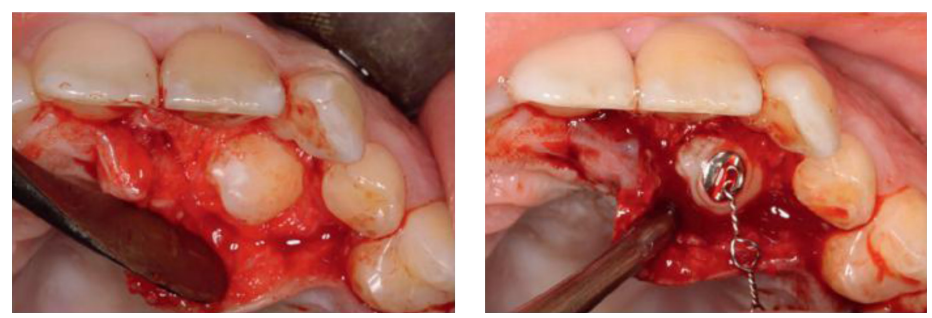

Figure 4

Example of eruption of palatal impacted canine. A flap is lifted and the tooth is exposed. The most delicate step is placing the canine for the bonding of the press stud. This is prepared in advance with a metal ligature strand punctuated with eyelets. The application of the self-etching solutions can be performed in just one application with a latency time before decreased adhesion (10 s). Moreover, it does not require any rinsing or drying of sources of bleeding. 

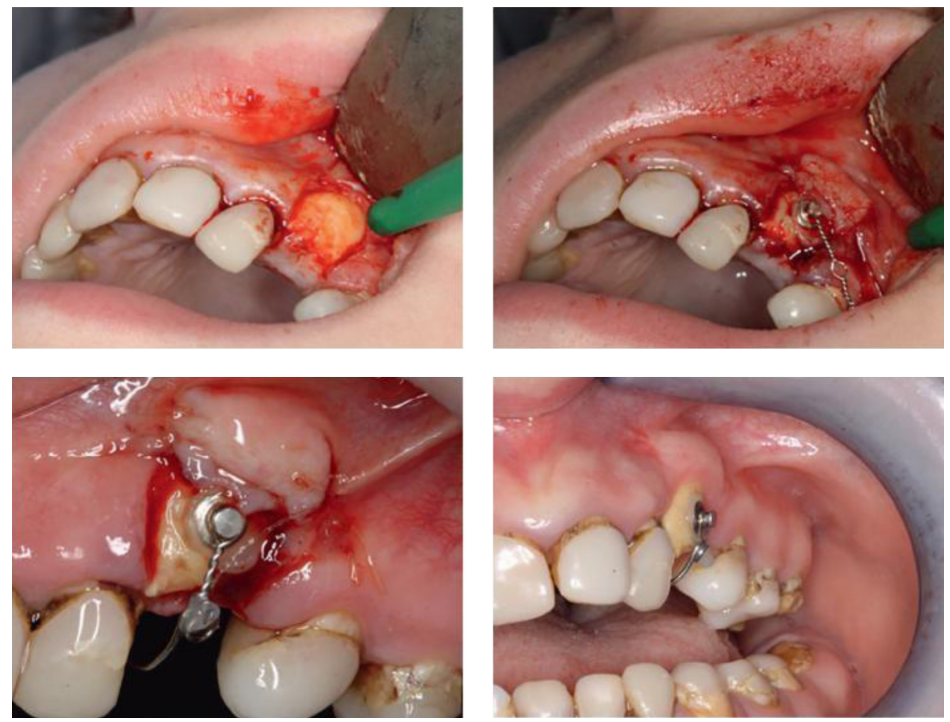

Figure 5

Setting up an impacted canine in an imperfect amelogenesis setting is a challenge. Here the vestibular canine has an apically moved flap. A traction button is bonded with a modified etching protocol (sodium hypochlorite). A connector is placed between the canine and two mini-screws inserted in palate. At 1 month after the procedure, note the healing of the displaced flap and keratinized gingiva gain at the canine neck.

It seems that the maturation stage of the canine does not affect the success rate of this treatment, but self-transplantation is only possible if $>2 / 3$ of the root is raised ${ }^{20}$. A strict protocol must therefore be followed: atraumatic extraction, flexible compression without immobilization of the tooth, and finally endodontic treatment, which should be performed within 14 days if the tooth is apexified or at the first signs of pulp necrosis if the apex is open.

However, the long-term success rate of transplanted teeth remains low. This solution should therefore be considered as a transitional solution for the maintenance of bone and periodontal volume before attempting a dental or implant reconstruction. This technique is probably not practiced because of the high rate of ankylosis that results in the grad- ual destruction of the root. As the tooth is replaced by bone, it does not compromise the placement of a future implant.

\section{Treatment, Orthodontic Stage}

There are usually two stages: preoperative and postoperative. At first, it may be useful to find the necessary place for the canine. This is done so as not to injure the teeth already present on the arch by putting them in contact with the impacted tooth? To do this, the use of three-dimensional examinations is necessary to plan dental movements ${ }^{39}$.

In adults, the rate of insertion after disimpaction is relatively random $(50 \%$ in patients aged $>30$ years). It may be preferable to start traction, without prior preparation, to overcome the failures due to possible ankylosis. 
In a second stage, the impacted canine will be moved to its normal position on the arch. Several types of anchorage can be used. In the first stage, place all the other teeth of the arcade. To do this, it is recommended to install a sufficiently rigid arch or a controlled sectoral mechanics ${ }^{9}$, to avoid an opening of the infraclusion due to the leveling of the archway ${ }^{11}$.

Other devices are described, in particular the use of bone anchors, transgingival ${ }^{10}$, or periosteal micro-screws. These include the traction devices described by Chillès ${ }^{12}$. These make it possible not to subject the arch to the stresses imposed by the traction of the canine. This is all the more important, as the risk of ankylosis could result in an ingression and a version of the adjacent teeth. Moreover, in some cases, these devices allow the traction of the canine without adhesion to the rest of the arch. The canine can then be brought as close as possible to its correct position. The multiattachment phase is then performed to obtain the perfect occlusion of the canine. However, this phase remains delicate, because it is the control of this tooth in three dimensions that will allow the placement of the canine (Fig. 6).
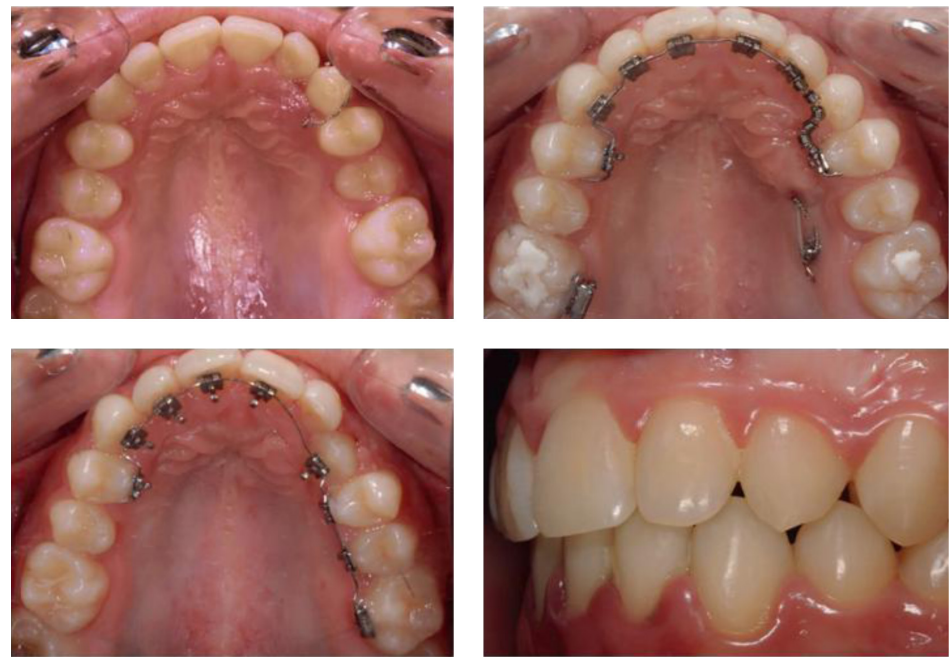

Figure 6

At first, the canine is removed by keeping the temporary tooth in place. The traction (purely distal) is done with a palatal screw. In the third step, the canine is set up on the arch using a multibrace palatal device.

\section{CONCLUSION}

This analysis of the literature, which includes the 2015 recommendations ${ }^{37}$, shows that, compared with those from
1968, techniques have improved. This is a matter of making our protocols more reliable, and they are constantly being 
improved. Today, it seems certain that the management of the impacted canine begins with the screening and interception phase. However, in cases requiring surgery, it should be noted that the treatment plan should be discussed by both the orthodontist and the surgeon. To repeat the conclusion of our authors: "The diversity of techniques clearly indicates the difficulty of impacted canine removal. However, all the authors quoted happily illustrated their case techniques. Finally, in spite of the progress of dental implantology, extraction should be limited to cases of refusal of treatment on the part of the patient, in the absence of a sign of root resorption of the neighboring teeth, in cases of satisfactory contact between the lateral incisors and the premolars, and in cases of very severe ectopia without any pathological signs.

Conflict of interest: The authors declare that they have no conflict of interest.

\section{BIBLIOGRAPHY}

1. Baccetti T. A controlled study of associated dental anomalies. Angle Orthod. 1998 Jun;68(3):267-74.

2. Becker A, Chaushu G, Chaushu S. Analysis of failure in the treatment of impacted maxillary canines. Am J Orthod Dentofacial Orthop. 2010 Jun;137(6):743-54.

3. Becker A, Chaushu S. Etiology of maxillary canine impaction: a review. Am J Orthod Dentofac Orthop Off Publ Am Assoc Orthod Its Const Soc Am Board Orthod. 2015 Oct;148(4):557-67.

4. Becker A, Chaushu S. Palatally impacted canines: the case for closed surgical exposure and immediate orthodontic traction. Am J Orthod Dentofac Orthop Off Publ Am Assoc Orthod Its Const Soc Am Board Orthod. 2013 Apr;143(4):451-9.

5. Becker A, Chaushu S. Success rate and duration of orthodontic treatment for adult patients with palatally impacted maxillary canines. Am J Orthod Dentofacial Orthop. 2003;124(5):509-14.

6. Becker A. In defense of the guidance theory of palatal canine displacement. Angle Orthod. 1995;65(2):95-8.

7. Becker A. Orthodontic treatment of impacted teeth. John Wiley \& Sons; 2012.

8. Bishara SE. Clinical management of impacted maxilary canines. Semin Orthod. 1998 Jun;4(2):87-98.

9. Bounoure GM, Frindel F. Désinclusion canine et thérapeutique bioprogressive. Rev Orthopédie Dento-Faciale. 1978 Jan;12(1):53-63.

10. Brézulier D, Sorel O. Les minivis: amélioration de la tenue, nouvelles perspectives. Rev Orthopédie Dento-Faciale. 2016 Jun;50(3):265-74.

11. Caldas SGFR, Ribeiro AA, Simplício H, Machado AW, Caldas SGFR, Ribeiro AA, et al. Segmented arch or continuous arch technique? A rational approach. Dent Press J Orthod. 2014 Apr;19(2):126-41.

12. Chillès $D$, Chillès J-G. Un dispositif à ancrage squeettique pour la traction et la mise en place des canines incluses. Rev Orthopédie Dento-Faciale. 2009 Mar;43(1):67-88. 
13. Dalessandri D, Migliorati M, Rubiano R, Visconti L, Contardo L, Di Lenarda R, et al. Reliability of a novel CBCT-based 3D classification system for maxillary canine impactions in orthodontics: the KPG index. Scientific World Journal. 2013;2013:921234.

14. Dalessandri D, Migliorati M, Visconti L, Contardo L, Kau CH, Martin C. KPG index versus OPG measurements: a comparison between 3D and 2D methods in predicting treatment duration and difficulty level for patients with impacted maxillary canines. BioMed Res Int. 2014;2014:537620.

15. Delsol L, Orti V, Chouvin M, Canal P. Canines et incisives maxillaires incluses. Diagnostic et traitement. Encyc Med Chir (Paris) 28-635-G-102008.

16. Ericson S, Kurol J. Radiographic examination of ectopically erupting maxillary canines. Am J Orthod Dentofac Orthop Off Publ Am Assoc Orthod Its Const Soc Am Board Orthod. 1987 Jun;91(6):483-92.

17. Ericson S, Kurol PJ. Resorption of incisors after ectopic eruption of maxillary canines: a CT study. Angle Orthod. 2000 Dec;70(6):415-23.

18. Fleury JE, Deboets D, Assaad-Auclair C, Maffre N, Sultan P. [The impacted canine. Review of 212 cases. General principles of treatment]. Rev Stomatol Chir Maxillofac. 1985;86(2):122-31.

19. Garcia R. Canine maxillaire absente: du diagnostic à la thérapeutique. Rev Orthopédie Dento-Faciale. 2010 Jun;44(2):63-82.

20. Gonnissen H, Politis C, Schepers S, Lambrichts I, Vrielinck L, Sun Y, et al. Long-term success and survival rates of autogenously transplanted canines. Oral Surg Oral Med Oral Pathol Oral Radiol Endod. 2010 Nov;110(5):570-8.

21. Husain J, Burden D, McSherry P, Morris D, Allen M, Clinical Standards Committee of the Faculty of Dental Surgery, Royal College of Surgeons of England. National clinical guidelines for management of the palatally ectopic maxillary canine. Br Dent J. 2012 Aug;213(4):171-6.

22. Jacoby H. The etiology of maxillary canine impactions. Am J Orthod. 1983 Aug;84(2):125-32.

23. Kau CH, Lee JJ, Souccar NM. The validation of a novel index assessing canine impactions. Eur J Dent. 2013 Oct;7(4):399-404.

24. Kau CH, Pan P, Gallerano RL, English JD. A novel 3D classification system for canine impactions--the KPG index. Int J Med Robot Comput Assist Surg MRCAS. 2009 Sep;5(3):291-6.

25. Kim Y, Hyun H-K, Jang K-T. Interrelationship between the position of impacted maxillary canines and the morphology of the maxilla. Am J Orthod Dentofac Orthop Off Publ Am Assoc Orthod Its Const Soc Am Board Orthod. 2012 May;141(5):556-62.

26. Kokich VG. Preorthodontic Uncovering and Autonomous Eruption of Palatally Impacted Maxillary Canines. Semin Orthod. 2010 Sep;16(3):205-11.

27. Kokich VG. Surgical and orthodontic management of impacted maxillary canines. Am J Orthod Dentofac Orthop Off Publ Am Assoc Orthod Its Const Soc Am Board Orthod. 2004 Sep;126(3):278-83.

28. Mathews DP, Kokich VG. Palatally impacted canines: the case for preorthodontic uncovering and autonomous eruption. Am J Orthod Dentofac Orthop Off Publ Am Assoc Orthod Its Const Soc Am Board Orthod. 2013 Apr;143(4):450-8.

29. McSherry PF. The ectopic maxillary canine: a review. Br J Orthod. 1998 Aug;25(3):209-16. 
30. Parkin N, Benson PE, Thind B, Shah A. Open versus closed surgical exposure of canine teeth that are displaced in the roof of the mouth. Cochrane Database Syst Rev. 2008 Oct 8;(4):CD006966.

31. Parkin NA, Milner RS, Deery C, Tinsley D, Smith A-M, Germain P, et al. Periodontal health of palatally displaced canines treated with open or closed surgical technique: a multicenter, randomized controlled trial. Am J Orthod Dentofac Orthop Off Publ Am Assoc Orthod Its Const Soc Am Board Orthod. 2013 Aug;144(2):176-84.

32. Peck S, Peck L, Kataja M. Concomitant occurrence of canine malposition and tooth agenesis: evidence of orofacial genetic fields. Am J Orthod Dentofac Orthop Off Publ Am Assoc Orthod Its Const Soc Am Board Orthod. 2002 Dec;122(6):657-60.

33. Peck $S$, Peck L, Kataja M. Prevalence of tooth agenesis and peg-shaped maxillary lateral incisor associated with palatally displaced canine (PDC) anomaly. Am J Orthod Dentofac Orthop Off Publ Am Assoc Orthod Its Const Soc Am Board Orthod. 1996 Oct;110(4):441-3.

34. Peck S, Peck L, Kataja M. The palatally displaced canine as a dental anomaly of genetic origin. Angle Orthod. 1994;64(4):249-56.

35. Peck S. Misleading article on palatally displaced canines. Am J Orthod Dentofac Orthop Off Publ Am Assoc Orthod Its Const Soc Am Board Orthod. 2016 Feb;149(2):149-50.

36. San Martín DE, English JD, Kau CH, Gallerano RL, McGrory KR, Salas AM, et al. The KPG index-a novel 3D classification system for maxillary canine impactions. Tex Dent J. 2012 Mar;129(3):265-74.

37. Société Française de Stomatologie, Chirurgie Maxillo-Faciale et Chirurgie Orale. Prise en charge d'une canine incluse, Recommandations de Bonne Pratique, Juillet 2015. 2015.

38. Sorel O, Glez D. Environnement parodontal des canines. Rev Orthopédie Dento-Faciale. 2010 Jun;44(2):199-214.

39. Sorel O, Ollivier A, Mano M-C. Intérêts de l'imagerie tridimensionnelle. Rev Orthopédie Dento-Faciale. 2010 Mar;44(1):83-98.

40. Vermette ME, Kokich VG, Kennedy DB. Uncovering labially impacted teeth: apically positioned flap and closed-eruption techniques. Angle Orthod. 1995;65(1):23-32; discussion 33.

41. Yan B, Sun Z, Fields H, Wang L, Luo L. Etiologic factors for buccal and palatal maxillary canine impaction: a perspective based on cone-beam computed tomography analyses. Am J Orthod Dentofac Orthop Off Publ Am Assoc Orthod Its Const Soc Am Board Orthod. 2013 Apr;143(4):527-34. 